\title{
Effects of non-contact electric fields on consolidation behavior of agglomerated yttria-stablized zirconia
}

\author{
Hasti Majidi and Klaus van Benthem
}

Department of Chemical Engineering and Materials Science, University of California, Davis, CA, 95616, USA

Electric field-assisted sintering (EFAS) techniques, which include spark plasma sintering and "flash sintering", have demonstrated the potential for enhanced densification at lower temperatures compared to conventional methods of sintering.[1-2] However, the fundamental mechanisms governing the enhancements of the densification processes are debated in the literature. The separate effects of the applied field and/or the resulting current remain unknown.[3] Studying the mechanisms of EFAS therefore requires separating the effects of the electric field from those of the electric current. Here, we report, for the first time, in situ scanning transmission electron microscopy (STEM) observations and quantitative shrinkage analysis of $3 \mathrm{~mol} \%$ yttria-stablized zirconia (3YSZ) powder agglomerates during consolidation in the presence of a non-contacting externally applied electric field.

3 YSZ particle agglomerates were dispersed in ethanol and drop-casted onto MEMS devices (Protochips, Inc.), which are capable of simultaneous heating and biasing. A schematic of an electrothermal MEMS device is illustrated in Figure 1. Agglomerates are supported by an amorphous carbon layer which covers the holey $\mathrm{SiC}$ membrane serving as a resistive heating element. A noncontacting electric field was applied by placing the 3YSZ agglomerates in between two parallel W electrodes under bias. Real-time STEM observations allow monitoring the evolution of particle agglomerates during in situ consolidation with and without applying the electric field. Figure 2 shows microstructural changes of two $3 \mathrm{YSZ}$ agglomerates during in situ isothermal heating at $900{ }^{\circ} \mathrm{C}$ in the absence (Figure 2(a-c)) and presence of an electric field (Figure 2(d-f)). In the absence of the electric field, the morphology of the agglomerate does not change significantly within 106 min at $900{ }^{\circ} \mathrm{C}$. However, when 3 YSZ agglomerates are exposed to $500 \mathrm{~V} / \mathrm{cm}$, neck formation and growth, particle coalescence and pore shrinkage is observed within only $4 \mathrm{~min}$ at $900{ }^{\circ} \mathrm{C}$. The shrinkage analysis of the agglomerate during in situ heating was performed using a recently developed image processing tool,[4] where the projected area of the agglomerate, obtained from STEM micrographs, is plotted as a function of time and electric field strength. While heating in the absence of the electric field slowly reduces the projected area of the agglomerate to $97 \%$ after $106 \mathrm{~min}$, the combination of an external electric field with sample heating leads to a sudden shrinkage of $93 \%$ only after 4 min at $900{ }^{\circ} \mathrm{C}$. Achieving similar magnitude of shrinkage without applied electric field requires higher temperatures and longer times (subsequent heating at $1000{ }^{\circ} \mathrm{C}$ for $28 \mathrm{~min}$ ). While the exposure of particle agglomerates to an electric field in the absence of current flow through the sample results in sudden consolidation, the magnitude of shrinkage is small compared to that observed during, e.g., flash sintering experiments. Hence, Joule heating due to raising electric currents must contribute significantly to densification during EFAS while effects of electric fields are appreciable. Future studies require the evaluation of defect formation as a function of electric field strengths to identify mechanisms of field-assisted sintering. 
[1] U. Anselmi-Tamburini et al, J. Mater. Res. 19 (2011), P. 3255.

[2] M. Cologna, A.L.G. Prette, and R. Raj, J. Am. Ceram. Soc. 94 (2011), P. 316.

[3] J.E. Garay, Annu. Rev. Mater. Res. 40 (2010), P. 445.

[4] H. Majidi, T. Holland, and K. van Benthem, Ultramicroscopy 152 (2015), P. 35.

[5] H. Majidi, and K. van Benthem (submitted for publication).

[6] This work was supported by the Army Research Office under award \#W911NF121049. The authors are grateful to Dr. John Damiano for useful discussions regarding Protochips MEMS devices.

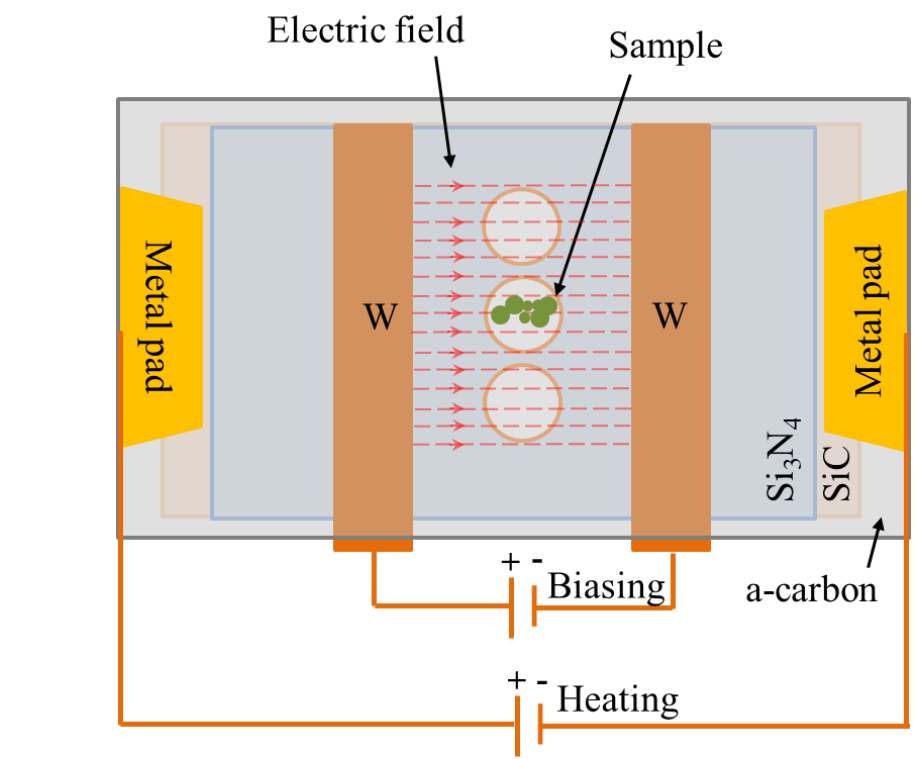

Figure 1: a schematic of electrothermal MEMS device enabling simultaneous heating and biasing to monitor particle agglomerates during in situ consolidation in the presence of non-contact electric field
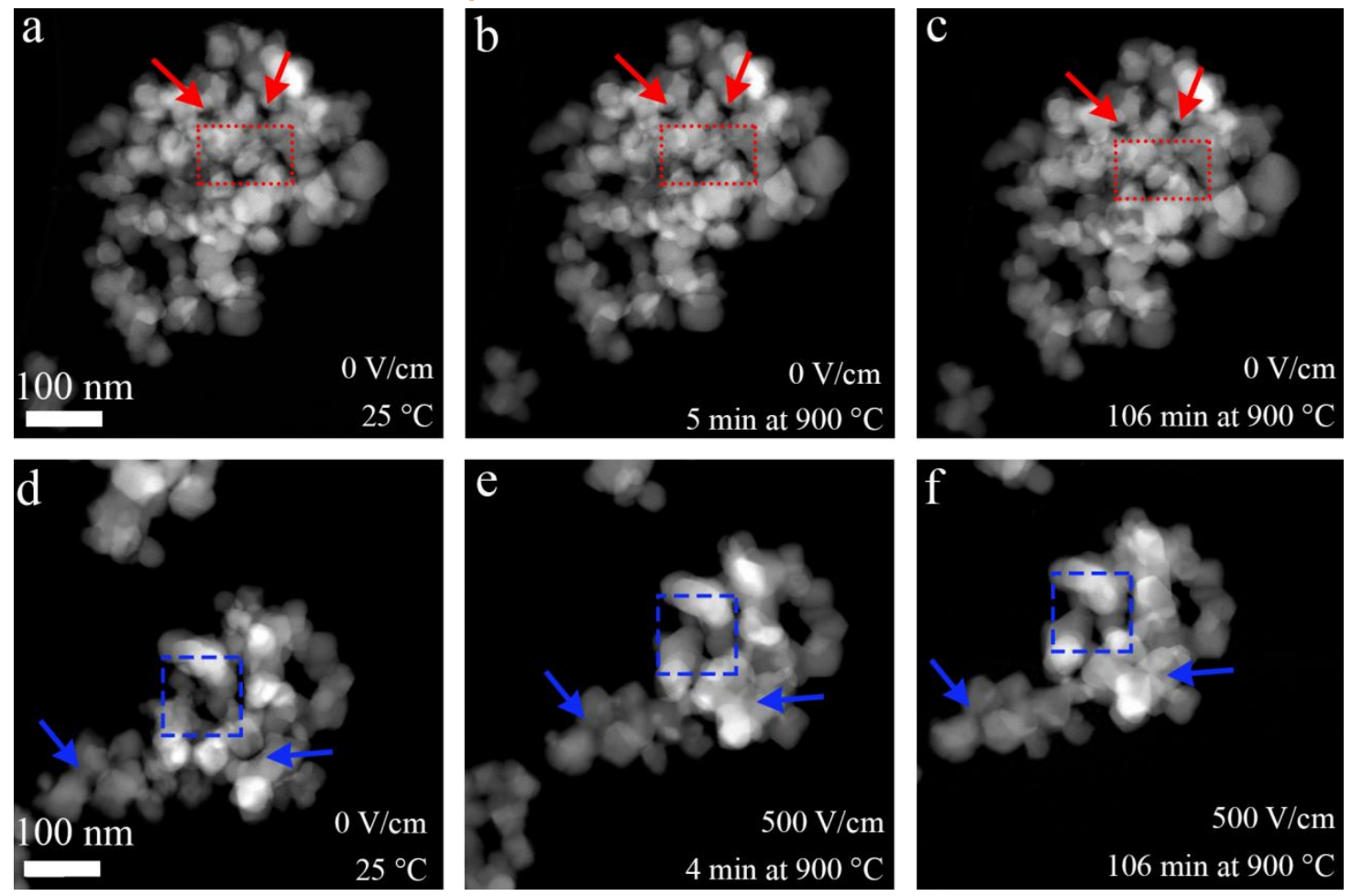

Figure 2: STEM micrographs of 3 YSZ agglomerates during in situ heating to $900{ }^{\circ} \mathrm{C}$ (a-c) in the absence and (d-f) presence of non-contact electric field. Data reproduced from [5]. 